\title{
Improvement of Beam-Quality Evaluation Method for Medical Linear Accelerator Using Magnetic Field
}

\author{
Jeongho Kim ${ }^{1}$, Manseok Han ${ }^{2 *}$, Sejong $\mathrm{Yoo}^{3}$, Kijin Kim ${ }^{3}$, and Jae-Hwan $\mathrm{Cho}^{4}$ \\ ${ }^{1}$ Department of Radiation Oncology, Konyang University Hospital \\ ${ }^{2}$ Department of Radiology, Kangwon National University \\ ${ }^{3}$ Department of Radiology, Konyang University Hospital \\ ${ }^{3}$ Department of Radiology, Konyang University Hospital \\ ${ }^{4}$ Department of International Radiological Science, Hallym University of Graduate Studies, Seoul 135-841, Korea
}

(Received 3 April 2015, Received in final form 15 June 2015, Accepted 15 June 2015)

\begin{abstract}
Beam-quality of medical linac evaluations vary by diverse factors. Because conventional beam-quality evaluation methods yield fragmentary results, a new beam-evaluation method is suggested, and its feasibility is evaluated. The PDDs (percentage depth doses) of 6 MV (Mega-voltage) and 10 MV photon, R (Range) of a 6 $\mathrm{MeV}$ (Mega Electron-voltage) and $9 \mathrm{MeV}$ electron were measured and compared with the conventional evaluation methods, and the improved methods $\mathrm{PDD}^{10}{ }_{5}, \mathrm{PDD}^{20}{ }_{10}, \mathrm{PDD}^{30}{ }_{20}, \mathrm{PDD}^{20}{ }_{5}, \mathrm{PDD}^{30}{ }_{10}$, and $\mathbf{R}^{70}{ }_{50}, \mathrm{R}^{50}{ }_{30}$, $R^{70}{ }_{30}$ as the magnetic field of the bending magnet was changed to $+2 \%$ to $-2 \%$, and the results were compared. The comparison showed that the improved methods exhibit a higher discrimination than the conventional methods in each energy regime. $\mathrm{PDD}_{5}^{10}, \mathrm{PDD}^{30}{ }_{20}, \mathrm{PDD}^{30}{ }_{10}$ and $\mathrm{R}^{70}{ }_{50}, \mathrm{R}^{50}{ }_{30}$ should be applied. These methods exhibit a higher discrimination in each energy regime than conventional beam-quality evaluation methods; therefore, they should be used for beam-quality evaluation according to the magnetic field variation.
\end{abstract}

Keywords : linac, beam quality, quality assurance, PDD, magnetic

\section{Introduction}

A medical linear accelerator is a radiation-generating apparatus for removing tumors in the body through precise emission of high-energy and high-dose radiation; therefore, its mechanical accuracy is very important $[1$, 2]. Radiation is emitted from a linear accelerator through a series of processes. First, thermal electrons are generated and discharged from the electron gun, and electrons with a certain amount of energy are accelerated in the accelerating tube under high-frequency waves that are released from the magnetron and klystron and delivered to the accelerating tube via a waveguide. The electrons accelerated in the accelerating tube by the high-frequency waves are delivered to the bending magnet, which bends or filters the energy of the electron beam. An electron accelerated in the accelerating tube does not always have a constant energy but rather exhibits a continuous spectrum

(C)The Korean Magnetics Society. All rights reserved.

*Corresponding author: Tel: +82-33-540-3383

Fax:+82-33-540-3389, e-mail: angio7896@naver.com distribution. At various velocities, its bending angle changes because of a constant magnetic field. The angle is bent to $270^{\circ}$ by the generally preset magnetic field, and energy is filtered by blocking the electrons whose angle deviates from the bending angle. Such energy filtering is applied within $\pm 1 \%$ of the target energy [3-5]. The responsible personnel at each hospital perform the quality assurance of the apparatus operation within this proper range under the guidelines of the International Atomic Energy Agency (IAEA) and Nuclear Safety and Security Commission (NSSC) [6, 7]. The quality assurance manager selects the quality assurance items according to the recommendations of the American Association of Physicists in Medicine for approval by the NSSC [8]. The quality assurance items suggested by American Association of Physicists in Medicine are divided into mechanical items, beam-measurement items, and safety items [9-12]. Among the beam-measurement items, the electronbeam quality is evaluated by $\mathrm{R}_{50}$ (range at $50 \%$ dose), and the photon-beam quality is evaluated by the $\mathrm{PDD}_{10}$ (percentage depth dose at $10 \mathrm{~cm}$ depth) with allowable errors of $1 \%$ and $1 \mathrm{~mm}$, respectively [13, 14]. However, 
the distribution of the beam quality can vary according to factors such as the variation in the magnetic field of the bending electromagnet, variation in the target position, and positional variation of the flux flatness filter or scattering foil. Among these, the variation in the magnetic field of the bending electromagnet directly affects the beam quality. However, conventional beam-quality evaluation methods cannot analyze this variation. Therefore, this study aimed to propose an improved beam-quality evaluation method to analyze the quality of a photon beam and an electron beam according to the magnetic field of the bending electromagnet and to evaluate the feasibility of this method.

\section{Materials and Method}

\subsection{Theory}

The radiation quality evaluation method proposed in this study was set up according to utilization of ratio, utilizing the relative dose ratio at a specific depth as shown in as formulas (1) and (2). Beam-Quality evaluation method in this experiment was set as using the ratio of relative dose at a constant depth so we set the equations (1), (2). A bending magnet is installed at the exit of the medical linear accelerator, and the variation of its magnetic field changes the rotation radius of the negatively charged electron beam [15-17]. This variation in the rotation radius manifests as a change in the beam quality when the position of the target or the electronbeam exit deviates. That is, the magnetic force changes according to the Biot-Savart Law, as indicated by Eqs. (3) and (4). The change in the rotational momentum of the electron beam is expressed by Eq. (5), and the amount of distortion of the rotation radius of the electron beam changes according to Eq. (6) $[18,19]$. As for deflection electromagnet of medical linear accelerator just like formula (3), change in the strength of the magnetic field is depending on permeability of the medium and distance and direction of a wire from an electric charge, current element, and external factors. But, since it cannot change geometric elements, it changes the current elements so as to change the strength of the magnetic field. And for incident velocity of an accelerated electron introduced as shown in formula (4) in connection to the change of magnetic field, a change in magnetic force is led to a change in the gyroradius. And the momentum of an electron is reflected as the momentum being generated by rotating and non-rotating wavelengths of radio waves, in the following formula (5). In other words, it is a change in the vector variation by the magnetic force and a change of variation in the momentum of the electron in progress as in formula (6). However, variation in the gyroradius being changed by such formula cannot reflect all the change factors by the energy filter and those in the radiation formation process according to the change in the energy distribution thus it cannot describe all the radiation quality variation. To this end, it was intended in this study to measure percentage depth dose according to the change of magnetic field and evaluated it in order to reflect the radiation quality variation as an integrated indicator.

Like the equation (3), In case of the bending electromagnet of LINAC, the magnetic field strength is changed by the direction and the distance between wire and current element, magnetic permeability and external factors. As geometric elements cannot be changed, we handled the current intensity. Like a equation (4), The change in the magnetic field lead the change of radius of rotation. and the momentum of the electrons with the wave length of radio waves and non-rotating wave is reflected like equation (5). In other words, the changes of vector and the momentum of electrons proceeds as in equation (6) However, This formula can't cover the change factors of energy filter and energy distribution so it can't indicate the change of beam quality for advisable index, we are to measure Percentage Depth Dose following the change of magnetic field.

$$
P D D_{y}^{x}=P D D_{x} / P D D_{y}
$$

$P D D_{y}^{x}$ : Ratio of Percentage Depth Dose at the depth of $\mathrm{x} \mathrm{cm}$ versus ratio of Percentage Depth Dose at $\mathrm{y} \mathrm{cm}$

$P D D_{x}$ : Percentage Depth Dose at the depth of $\mathrm{x} \mathrm{cm}$

$P D D_{x}$ : Percentage Depth Dose at the depth of $\mathrm{y} \mathrm{cm}$

$R_{\beta}^{\alpha}=R_{\alpha} / R_{\beta}$

$R_{\beta}^{\alpha}$ : Ratio of the depth of Percentage Depth Dose of $\alpha$ $\%$ against Percentage Depth Dose of $\beta \%$

$R_{\alpha}$ : Depth of Percentage Depth Dose of $\alpha \%$

$R_{\beta}$ : Depth of Percentage Depth Dose of $\beta \%$

$B=\frac{\mu_{0}}{4 \pi} \int \frac{I d l_{\overrightarrow{ }}}{r^{2}} C$

$F=q_{1} v B=\frac{\mu_{0}}{4 \pi} q_{1} v \int \frac{I d l_{\ni}}{r^{2}} C$

$B$ : power of magnetic field

$\mu_{0}$ : magnetic permeability of a material

Idl : current element

$r$ : distance between the conducting wire and charge

$q_{1}:$ incident charge

$\vec{r}:$ r-directional unit vector

$C:$ cross product

$F$ : magnetic force 
$v:$ speed of incident charge

$$
h v=h v\left(1-\frac{r_{0}}{r}\right)+h v \frac{r_{0}}{r}
$$

$h:$ Planck's constant

$v:$ wave

$r:$ radius of rotational wave

$r_{0}:$ radius of non-rotational wave

$$
\vec{x}=\sqrt{\left(r^{2}\right)+\left(f^{2}\right)}
$$

$\vec{x}_{\vec{f}}$ : vector of rotation radius variation

$\vec{f}$ : force vector between the bending magnet and electron

\subsection{Target}

The linear accelerator CLINAC-iX (Varian, USA) and the bending magnet installed inside were investigated. For beam-dose measurements, a DOSE-1 electrometer (IBA, Germany), a Blue Phantom ${ }^{2}$ (IBA, Germany), an Ionization Chamber-FC23-C (IBA, Germany), and an Ionization Chamber-PPC40 (IBA, Germany) were used (Fig. 1). The beam-quality evaluation index was based on the existing photon-beam evaluation index $\mathrm{PDD}_{10}$ and the existing electron-beam evaluation index $\mathrm{R}_{50}$. The improved evaluation indices $\mathrm{PDD}^{10}{ }_{5}, \mathrm{PDD}^{20}{ }_{10}, \mathrm{PDD}^{30}{ }_{20}, \mathrm{PDD}^{20}{ }_{5}$, and $\mathrm{PDD}^{30}{ }_{10}$ were applied to the photon beam, and $\mathrm{R}^{70}$,
$\mathrm{R}^{50}{ }_{30}$, and $\mathrm{R}^{70}$ were applied to the electron beam.

\subsection{Method}

In the experiment, the magnetic field of the bending magnet in the linear accelerator was divided into a low magnetic field, which is lower than the standard magnetic field by $-1 \%$ and $-2 \%$, and high magnetic fields, which are higher than the standard magnetic field by $+1 \%$ and $+2 \%$. The strength of magnetic field in bending magnet is 135 Gauss at $4 \mathrm{~mA}$ for $6 \mathrm{MV}$ photon beam, whereas it is 226 Gauss at $6.7 \mathrm{~mA}$ for $10 \mathrm{MV}$ photon beam. And for $6 \mathrm{MV}$ electron beam, it is 44 Gauss at $1.3 \mathrm{~mA}$ whereas it is 68 Gauss at $2 \mathrm{~mA}$. Although the change in the magnetic field should be measured by increasing the applied voltage, it was measured as the increase in the magnetic field caused by the change in the applied voltage according to the degree of vacuum in the bending magnet. The PDDs of the $6 \mathrm{MV}$ photon beam, $10 \mathrm{MV}$ photon beam, 6 $\mathrm{MeV}$ electron beam, and $9 \mathrm{MeV}$ electron beam were measured for five conditions. In the PDD measurement of the photon beams, the Blue Phantom was filled to $40 \mathrm{~cm}$ or higher with distilled water, and the horizontal levels of the base plate and water tank were adjusted. The FC23-C ion chamber was placed at the center of the water tank, and its radiation dose was measured as gradually moving to $30 \mathrm{~cm}$ from the surface. The irradiation conditions were as follows: the angles of the gantry and collimator
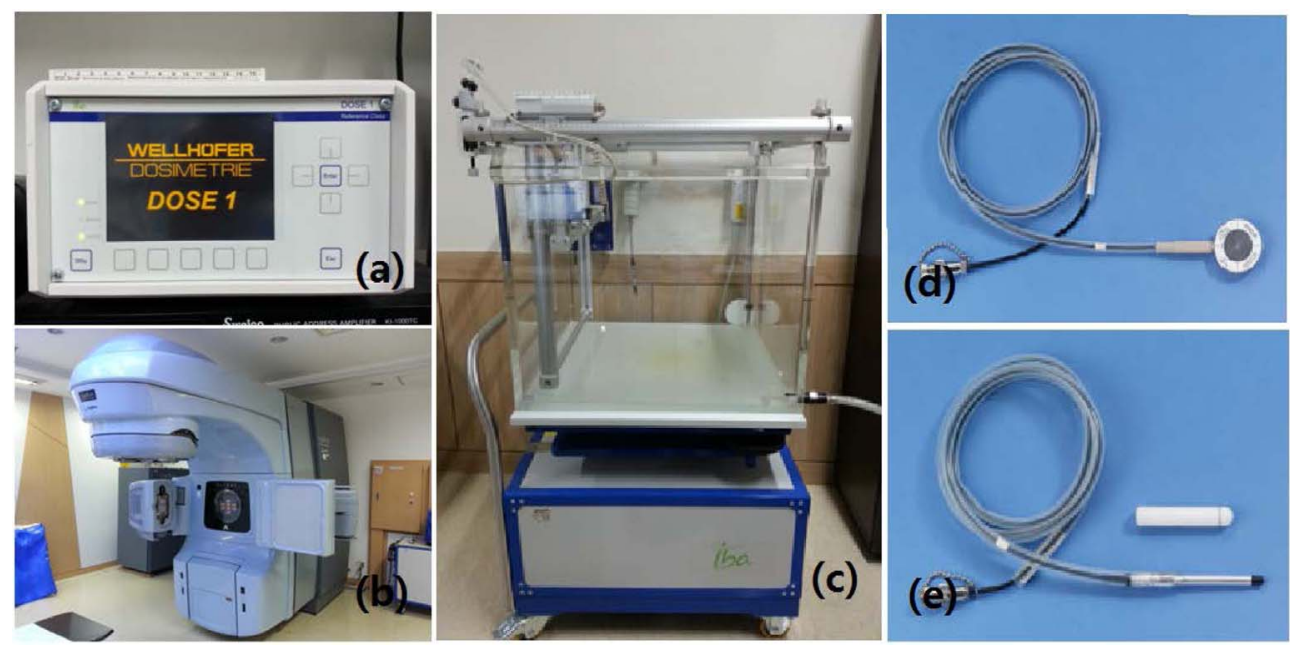

Fig. 1. (Color online) (a) Potentiometer that displays a current applied to the ionization chamber used in the radiation measurement by quantifying it. For the applicable potentiometer, calibration is to be carried out once a year together with the ionization chamber. (b) It is an equipment being used to move the position of the ionization chamber remotely, as a water phantom being used for the dose measurement of a radiation therapy equipment by filling the chamber with water that owns the electron density similar to the human body (c) It is an equipment which is used in radiotherapy as a linear accelerator for medical use, and it can irradiate photon beam and electron beam mounted with a posture photography device and a multi-leaf collimator (d) It is a Parallel Plate type ionization chamber being used to measure electron beam of a linear accelerator for medical use. It is made of Graphite and PMMA and its active volume is $0.4 \mathrm{cc}$ (e) It is a Farmer type ionization chamber being used to measure photon beam of a linear accelerator for medical use. It is made of Shonka C552 and its active volume is $0.23 \mathrm{cc}$. 


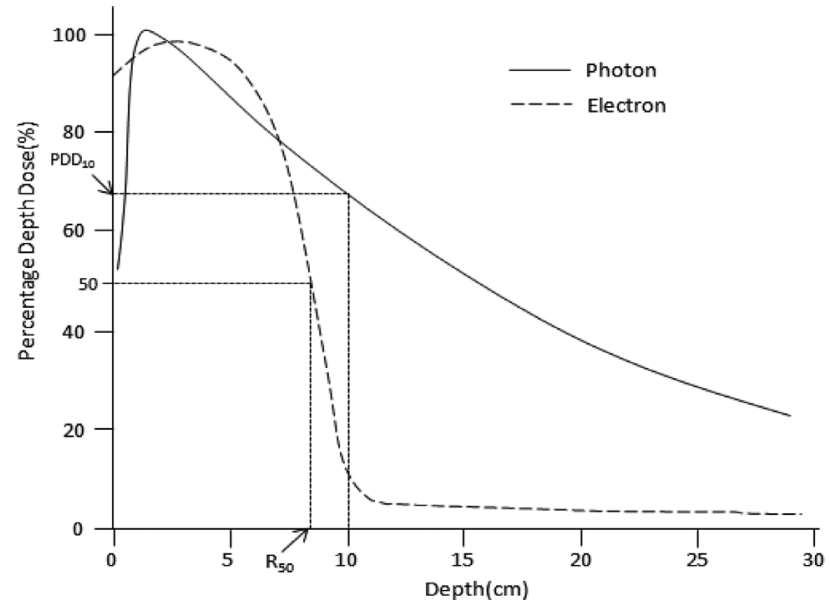

Fig. 2. The graph of Relative dose percentage for photon beam shows deeper maximum dose point and lower rate of dose reduction, whereas the graph of Relative dose percentage for electron beam shows shallower maximum dose point and higher rate of dose reduction. The beam quality evaluation indices are based on the relative dose percentage at the depth of $10 \mathrm{~cm}$ for photon beam whereas it is based on the length for $50 \%$ of dose for electron beam. As the index value varies depending on the different linear accelerators for medical use, the measurement is carried out at the initial installation. As for the applicable linear accelerator, the photon beam quality index values was measured as $67.74 \%$, while the electron beam quality index values was measured as $8.4 \mathrm{~cm}$. were set to $0^{\circ}$, and the radiation field was set to 10 by 10 $\mathrm{cm}^{2}$. The measured ionization amount was converted into doses by the electrometer. The PDDs of the electron beam were measured by mounting PPC40 to the $30 \mathrm{~cm}$ Solid Phantom and moving it at intervals of $0.5 \mathrm{~cm}$ from the surface. The irradiation conditions were as follows: the angles of the gantry and collimator were set to $0^{\circ}$, and the electron beam applicator used 10 cones. As in the PDD measurement of the photon beam, the measured ionization amount was converted into doses by the electrometer and additionally smoothed. The PDD measurements obtained under each condition were subjected to the conventional beam-quality method and improved beamquality method, and then they were compared. The standard-condition result and the improved-condition result were compared with regard to the percentage change and percentage-change increase (Figs. 2, 3). The exposure dose varies depending on temperature, humidity and pressure, however, since percentage depth dose was used in this study rather than the exposure dose, it did not consider such as temperature, humidity and pressure during measurement. But calibration is carried out once a year by an officially certified agency for the ionization chamber and the potentiometer in compliance with the regulations.

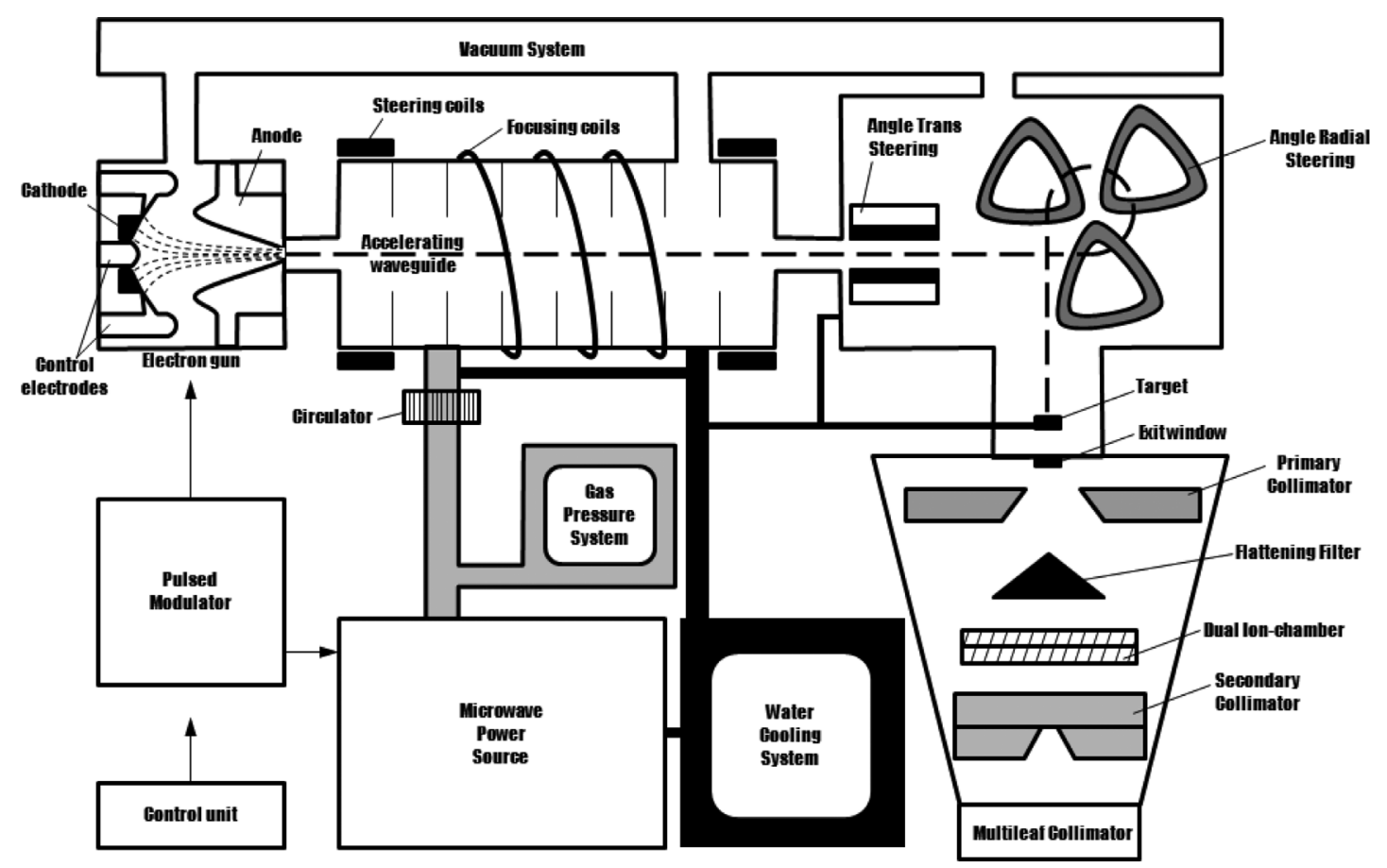

Fig. 3. The structure of a linear accelerator for medical use can be described as simple as shown in the figure. The beam flows into the acceleration pipe where the electron beam is generated in an electron gun and projected from the gun, There, the beam is accelerated by radio waves or bent and energy filtered via the bending magnet. There of the radiation beam formed through a collimator and a filter is to be irradiated finally. Also, according to the change in the magnetic field of Bending Magnet, the bending angle and the quality of radiation beam is to be changed. 


\section{Result}

\subsection{Photon Beam}

The PDDs of the $6 \mathrm{MV}$ photon beam and $10 \mathrm{MV}$ photon beam are shown in Figs. 4 and 5, respectively, and the beam-quality results are given in Table 1 .

\subsection{Electron Beam}

The PDDs of the $6 \mathrm{MeV}$ electron beam and $9 \mathrm{MeV}$ electron beam are shown in Figs. 6 and 7, respectively,

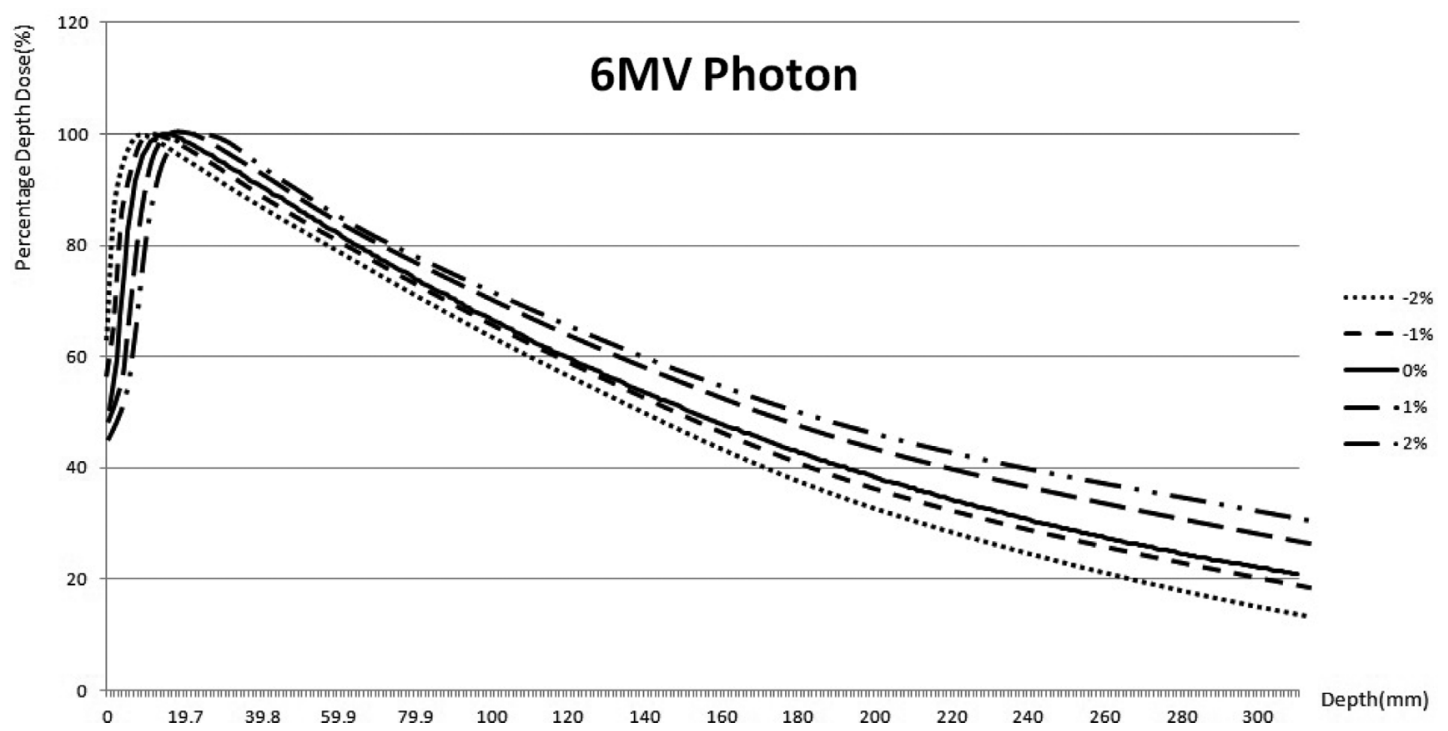

Fig. 4. When investigated $6 \mathrm{MV}$ photon beam of a linear accelerator for medical use, the ratio of dose value according to the depth in the water phantom was referred as Percentage Depth Dose and the Percentage Depth Dose was measured as changing the strength of magnetic field to $-2 \%,-1 \%,+1 \%$ and $+2 \%$. As the results, it was identified that the smaller the strength of magnetic field was, the deeper maximum dose depth gets, and the decrease of Relative dose percentage gets bigger. On the contrary, the larger the strength of magnetic field was, the deeper the maximum dose gets and the reduction of Relative dose percentage gets smaller. The reason for aforementioned changes is because as the strength of magnetic field is smaller, it is distributed more to the lower energy side while the strength of magnetic field is bigger, it is distributed more to the higher energy side.

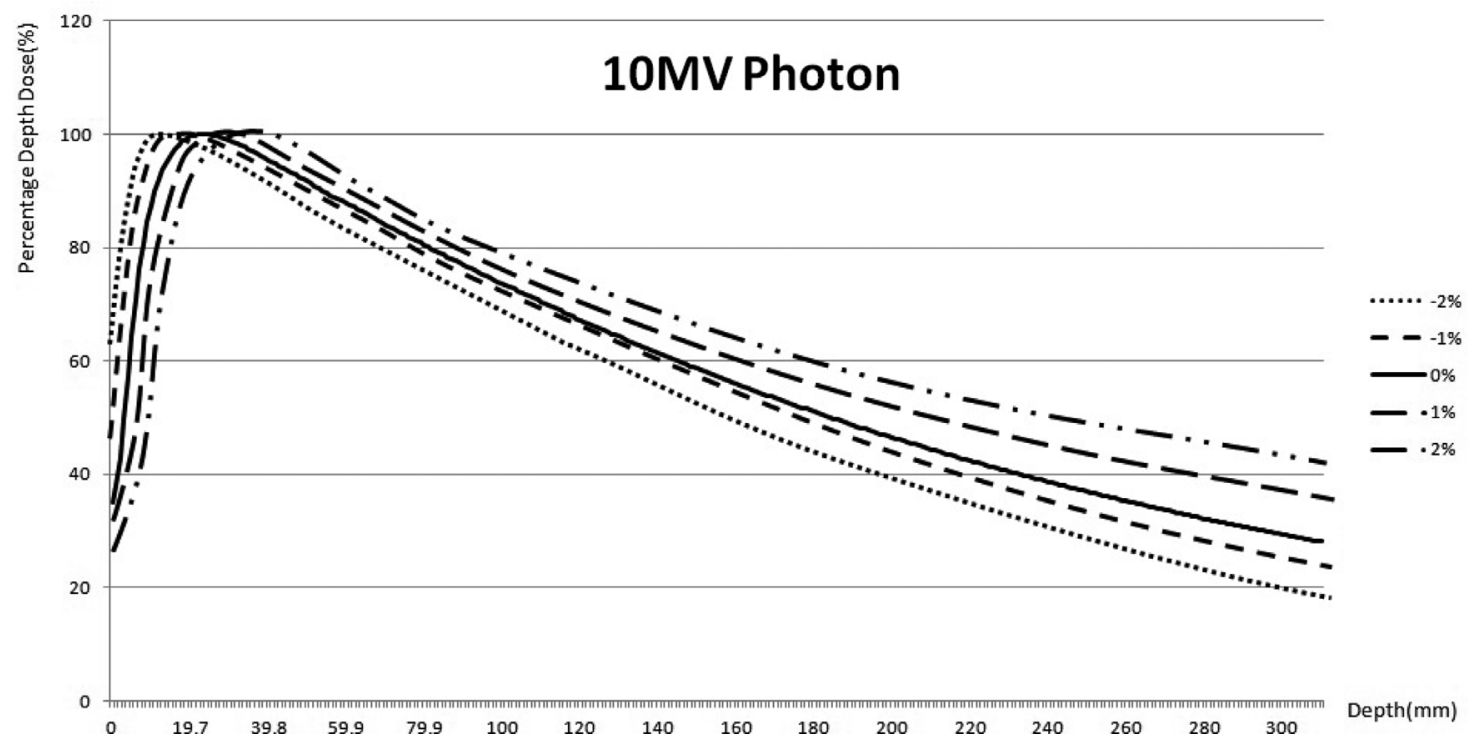

Fig. 5. 10MV photon beam exhibits its characteristics similar to those of $6 \mathrm{MV}$ photon beam. This is also because the strength of magnetic field is presented as proportional to the energy distribution. But, it should be noted that as higher as the energy level is, the depth-dependent difference gap of Percentage Depth Dose value gets wider. 


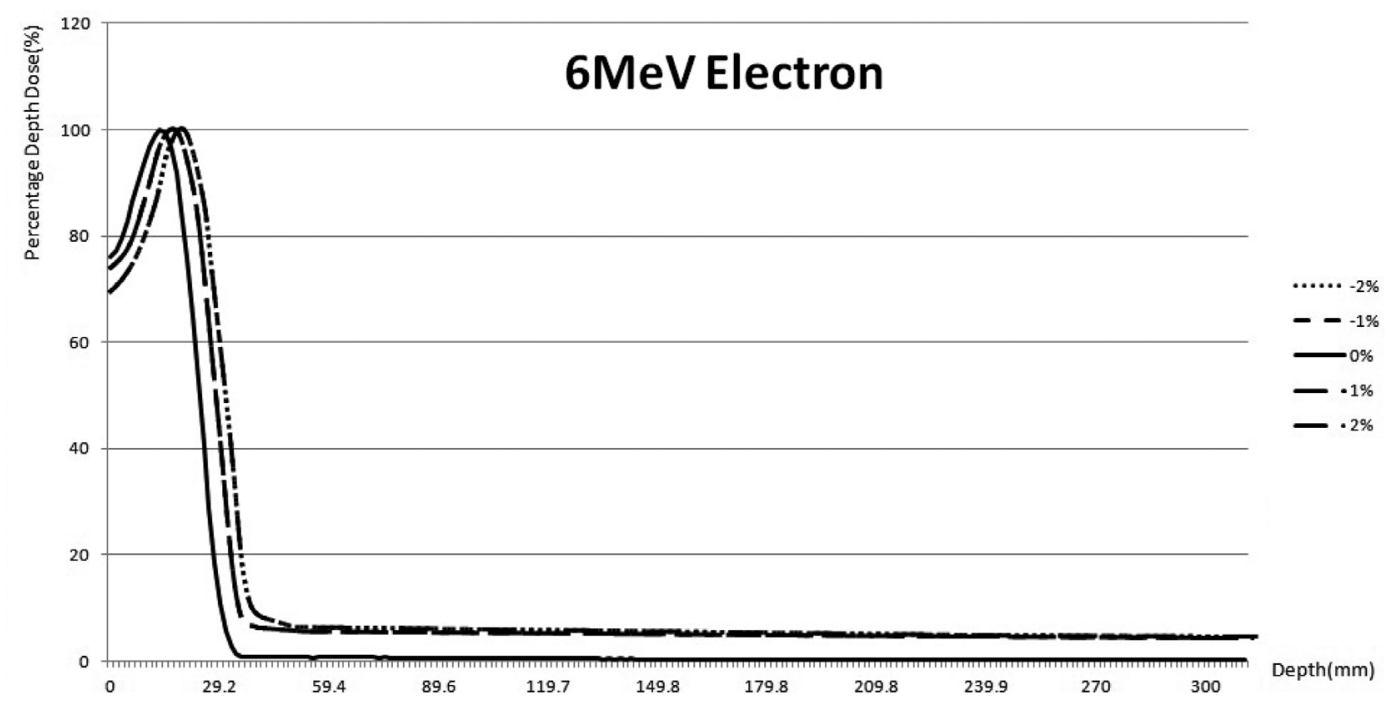

Fig. 6. When investigated $6 \mathrm{MV}$ electron beam of a linear accelerator for medical use, the Percentage Depth Dose was measured as changing the strength of magnetic field to $-2 \%,-1 \%,+1 \%$ and $+2 \%$. As the results, it was identified that there was nearly no variation by the increase/decrease of the strength of magnetic field, but it was changed according to the absolute values of variation. As the strength of magnetic field got changed, the maximum dose gets deeper and the tail side formed by Photon contamination was distributed to the high doses. This is a change due to the deviation range from the central axial radiation flux but does not depend on the directionality And it is a phenomenon emerged by the characteristics of electron in the beam due to the relative filtering of electron beam in the low energy domain.

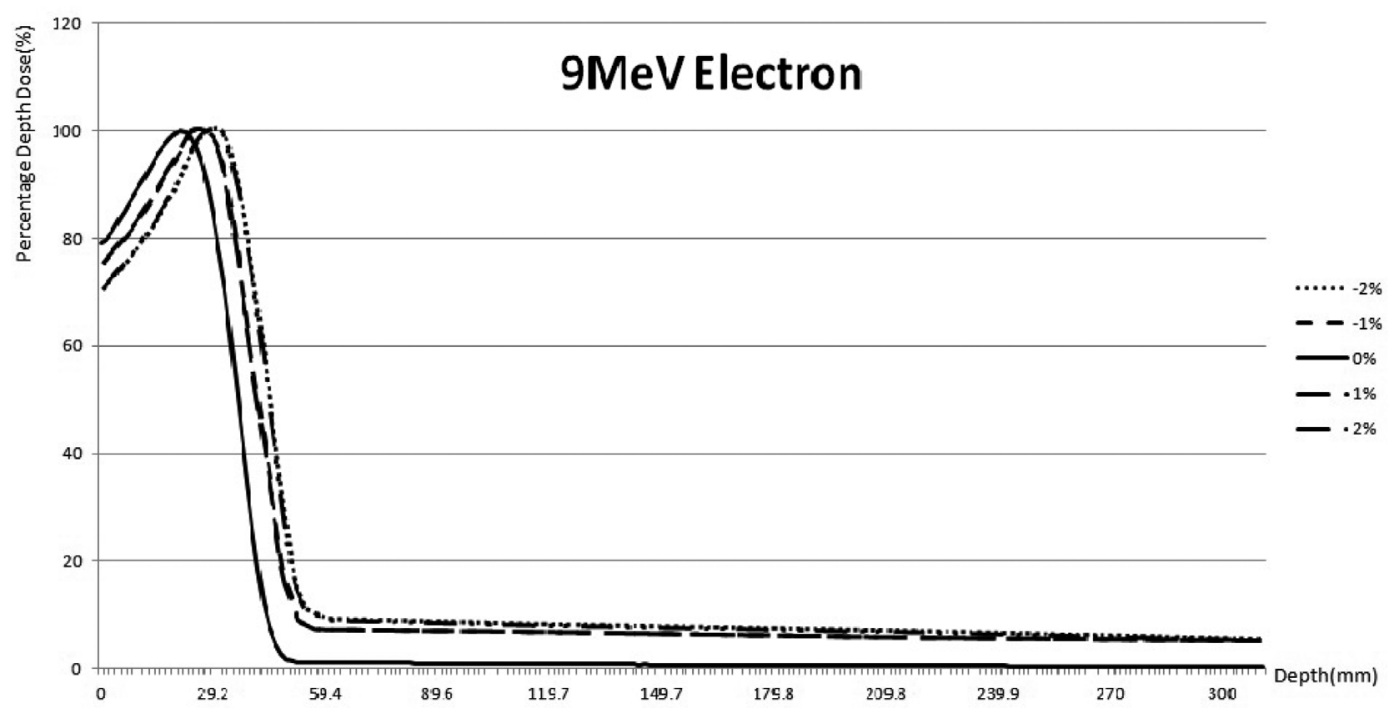

Fig. 7. $9 \mathrm{MeV}$ electron beam exhibits features similar to $6 \mathrm{MeV}$ electron beam. This is also because the strength of magnetic field and the energy distribution are presented in proportional manner. But, it should be noted that as higher as the energy level is, the depth-dependent difference gap of Percentage Depth Dose value gets wider.

and the beam-quality results are given in Table 2 .

\section{Discussion}

The beam quality of a medical linear accelerator is generally evaluated with regard to the relative dose rate under the assumption of a uniform variation of the energy distribution in accordance with the guidelines of the American Association of Physicists in Medicine. However, various factors affect the beam quality, and these differ between photon beams and electron beams [20]. Therefore, the beam quality should be evaluated from various 
Table 1. This table shows existing and proposed beam quality index values of $6 \mathrm{MeV}$ electron beam and $9 \mathrm{MeV}$ electron beam. In case of an existing beam quality index value $\mathrm{R}_{50}$, it indicates the depth, presenting the value of depth unlike the proposed index value as percentage ratio. According to the change of energy distribution, $\mathrm{R}^{70}{ }_{50}$ and $\mathrm{R}^{50}{ }_{30}$ have which are the ratio values for low dose or high dose have higher discrimination ability compared to R50, which displays a single point value.

\begin{tabular}{|c|c|c|c|c|c|c|}
\hline Index & Magnetic Field & $-2 \%$ & $-1 \%$ & $0 \%$ & $+1 \%$ & $+2 \%$ \\
\hline \multirow{2}{*}{$\mathrm{R}_{50}$} & $6 \mathrm{MeV}$ & 28.99153 & 26.72632 & 23.53197 & 26.69854 & 28.89244 \\
\hline & $9 \mathrm{MeV}$ & 40.11870 & 37.70021 & 34.8053 & 37.68995 & 40.13387 \\
\hline \multirow{2}{*}{$\mathrm{R}^{70}{ }_{50}$} & $6 \mathrm{MeV}$ & 0.913705 & 0.905096 & 0.892049 & 0.907323 & 0.917235 \\
\hline & $9 \mathrm{MeV}$ & 0.940594 & 0.932482 & 0.926982 & 0.930721 & 0.941737 \\
\hline \multirow{2}{*}{$\mathrm{R}^{50}{ }_{30}$} & $6 \mathrm{MeV}$ & 0.932177 & 0.907789 & 0.892537 & 0.904787 & 0.929056 \\
\hline & $9 \mathrm{MeV}$ & 0.954192 & 0.942455 & 0.928632 & 0.942515 & 0.955348 \\
\hline \multirow{2}{*}{$\mathrm{R}_{30}^{70}$} & $6 \mathrm{MeV}$ & 0.851735 & 0.821636 & 0.796187 & 0.820935 & 0.852163 \\
\hline & $9 \mathrm{MeV}$ & 0.897507 & 0.878822 & 0.860825 & 0.877219 & 0.899686 \\
\hline
\end{tabular}

Table 2. This table shows existing and proposed beam quality index values of $6 \mathrm{MeV}$ photon beam and $10 \mathrm{MeV}$ photon beam. In case of an existing beam quality index value, it only presents the value of Percentage Depth Dose against the single depth. In comparison, the proposed index value presents the relative values against low dose and high dose. In case of test value, when the strength of magnetic field is decreased, the index value of $\mathrm{PDD}^{10}{ }_{5}$ shows discrimination ability, whereas when the strength of magnetic field is increased, the index values of $\mathrm{R}^{70}{ }_{50}$ and $\mathrm{R}^{50}{ }_{30}$ present discrimination ability.

\begin{tabular}{|c|c|c|c|c|c|c|}
\hline Index & Magnetic Field & $-2 \%$ & $-1 \%$ & $0 \%$ & $+1 \%$ & $+2 \%$ \\
\hline \multirow[b]{2}{*}{$\mathrm{PDD}_{10}$} & $6 \mathrm{MV}$ & 62.0 & 65.2 & 66.7 & 68.0 & 70.0 \\
\hline & $10 \mathrm{MV}$ & 68.1 & 70.1 & 73.7 & 75.8 & 78.5 \\
\hline \multirow{2}{*}{$\mathrm{PDD}^{10}{ }_{5}$} & $6 \mathrm{MV}$ & 0.753341 & 0.763466 & 0.772885 & 0.779817 & 0.789177 \\
\hline & $10 \mathrm{MV}$ & 0.781860 & 0.779755 & 0.802832 & 0.814178 & 0.823715 \\
\hline \multirow{2}{*}{$\mathrm{PDD}^{20}{ }_{10}$} & $6 \mathrm{MV}$ & 0.487097 & 0.527607 & 0.574213 & 0.619118 & 0.641429 \\
\hline & $10 \mathrm{MV}$ & 0.569750 & 0.600571 & 0.630936 & 0.680739 & 0.704459 \\
\hline \multirow{2}{*}{$\mathrm{PDD}^{30}{ }_{20}$} & $6 \mathrm{MV}$ & 0.437086 & 0.500000 & 0.574413 & 0.636580 & 0.685969 \\
\hline & $10 \mathrm{MV}$ & 0.458763 & 0.551069 & 0.632258 & 0.707364 & 0.752260 \\
\hline \multirow{2}{*}{$\mathrm{PDD}^{20}{ }_{5}$} & $6 \mathrm{MV}$ & 0.366950 & 0.402810 & 0.443801 & 0.482798 & 0.506201 \\
\hline & $10 \mathrm{MV}$ & 0.445465 & 0.468298 & 0.506536 & 0.554243 & 0.580273 \\
\hline \multirow{2}{*}{$\mathrm{PDD}^{30}{ }_{10}$} & $6 \mathrm{MV}$ & 0.212903 & 0.263804 & 0.329835 & 0.394118 & 0.440000 \\
\hline & $10 \mathrm{MV}$ & 0.261380 & 0.330956 & 0.398915 & 0.481530 & 0.529936 \\
\hline
\end{tabular}

perspectives, rather than simply by applying the aforementioned evaluation methods. Accordingly, the present study aimed to propose a beam-quality evaluation method for each range according to the magnetic field of the bending magnet, in order to evaluate the usability of the conventional beam-quality methods and select an efficient method. The conventional beam-quality evaluation method was compared with the beam-quality evaluation method based on the relative dose rate. For the photon beam, the peak dose on the PDD curve moved toward the surface as the magnetic field decreased, and the relative dose rate decreased as the depth increased. This change in the PDD curve can be explained by the distribution of the photon beam on the side of the accelerating tube of the flattening filter with the diminishing magnetic field of the bending magnet and the consequent increase in the rotation radius and by the movement of the focus point of the collected electrons from the target position to the flattening filter. Moreover, the peak radiation-dose point moved away from the surface as the magnetic field increased, and the relative dose increased with the depth. The change in the PDD curve is explained by the distribution of the photon beam on the opposite side of the accelerating tube of the flattening filter with the decreasing rotation radius, due to the increasing magnetic field of the bending magnet and the movement of the focus point of the collected electrons to the prior target position. In comparison, the PDD curve of the electron beam exhibited a similar dose distribution 
for a low magnetic field and a high magnetic field. This is because there are no effects on the electron beam from the target or the flattening filter, compared with the photon beam. Furthermore, the position transfer of the distribution is similar symmetrically, depending on the reduction of the rotation radius for a high magnetic field and the increase in the rotation radius for a low magnetic field. The transfer distance is proportional to the change in the magnetic field. When the beam-quality index on the PDD curve based on the change in the magnetic field of the bending magnet is used, the conventional method and the improved method exhibit a difference. Because the conventional beam-quality evaluation method can evaluate a photon beam only at the depth of $10 \mathrm{~cm}$ in depth from the surface of water, it has difficulty reflecting the energydistribution effect and expressing the index error rate according to the change in the magnetic field. The improved beam-quality method can effectively express the error rate using the relative rate and can reflect the energy-distribution effect. Furthermore, when the improved beam-quality evaluation method was applied to the electron beam, it exhibited a greater expression and an easier energy distribution analysis, as it did for the photon beam. Therefore, instead of the conventional beam-quality method, an improved method that considers various factors should be applied to evaluate the beam quality of a medical accelerator. However, even the improved method will have difficulties in evaluating the beam quality around the peak dose point, where there is a large change in the beam quality. Therefore, the change in the beam quality should be checked up to a depth of $5 \mathrm{~cm}$ for 1-field radiation treatment. Moreover, for an electron beam, the magnetic field changes symmetrically. Therefore, a beam-quality evaluation method that can account for the symmetry in the beam-quality method applied for 1-field radiation treatment and in electron beam-quality evaluation must be developed.

\section{Conclusion}

Conventional beam-quality evaluation methods were limited to fragmentary evaluations. In particular, a separate evaluation index was required to analyze the change in the beam quality according to the magnetic field of the bending magnet in a linear accelerator. This study proposed $\mathrm{PDD}^{10}{ }_{5}, \mathrm{PDD}^{20}{ }_{10}, \mathrm{PDD}^{30}{ }_{20}, \mathrm{PDD}^{20}{ }_{5}$, and $\mathrm{PDD}^{30}{ }_{10}$ for the photon beam and $\mathrm{R}_{50}^{70}, \mathrm{R}^{50}{ }_{30}$, and $\mathrm{R}^{70}{ }_{30}$ for the electron beam. The magnetic field was changed from $+2 \%$ to $-2 \%$ in intervals of $1 \%$ to compare the index values. The results showed that $\mathrm{PDD}^{10}{ }_{5}, \mathrm{PDD}^{30}{ }_{20}$, and $\mathrm{PDD}^{30}{ }_{10}$ exhibited a relatively high discrimination com- pared with the conventional index for the photon beam, and $\mathrm{R}_{50}^{70}$ and $\mathrm{R}_{30}^{50}$ exhibited a high discrimination according to the energy ranges for the electron beam. These results indicate that in future beam-quality evaluations according to the magnetic field of the bending magnet in the medical accelerator, $\mathrm{PDD}_{5}^{10}, \mathrm{PDD}^{30}{ }_{20}$, and $\mathrm{PDD}^{30}{ }_{10}$ should be applied for the photon beam and $\mathrm{R}^{70}{ }_{50}$ and $\mathrm{R}^{50}{ }_{30}$ for the electron beam. Furthermore, for an absolute beamquality evaluation in the future, a separate measurement device should be developed for evaluations according to the ranges of the magnetic-field variation.

\section{References}

[1] C. Z. Kazmark, International Journal of Radiation Oncology, Biology, Physics 13, 1599 (1987).

[2] American Association of Physicists in Medicine, Physical Aspects of Quality Assurance in Radiation Therapy 13 (1984).

[3] S. E. Griffiths, C. A. Short, C. S. Jackson, and D. Ash, Radiotherapy: Principles to Practice, Churchill Livingstone, London (1995) pp. 27-88.

[4] S. S. Kang, I. H. Ko, and G. J. Kim, Radiation Therapeutics, Chung-gu munhwasa, Seoul (2014) pp. 63-98.

[5] F. M. Khan, The Physics of Radiation Therapy, Wolters Kluwer, Toronto (2010) pp. 132-211.

[6] Korea Food \& Drug Administration, Quality assurance for dosimetry in radiotherapy (2007).

[7] Korea Institute of Nuclear Safety, The Survey on the Status of Nuclear Industries 5 (1999).

[8] Ministry of Science and Technology, Development of 3Dimensional Dosimetry Technique for Therapeutic Radiation (2012).

[9] R. N. Lee, S. J. Lee, and J. H. Choi, Medical Physics 14, 20 (2003).

[10] D. Baltas, K. Geramani, G. T. Ioannidis, K. Hierholz, B. Rogge, C. Kolotas, K. Müller-Sievers, N. Milickovic, B. Kober, and N. Zamboglou, International Journal of Radiation Oncology, Biology, Physics 43, 653 (1999).

[11] K. H. Cho, S. H. Lee, J. H. Choi, D. O. Shin, J. S. Jang, C. K. Min, D. H. Choi, Y. H. Kim, E. S. Kim, and S. I. Kwon, 2006 Korean Society of Medical Physics Spring Conference, 3469 (2006).

[12] H. D. Huh, S. H. Kim, S. J. Cho, D. O. Shin, S. I. Kwon, H. J. Kim, W. C. Kim, and J. J. Loh, The Journal of the Korean Society for Therapeutic Radiology and Oncology 4, 23 (2005).

[13] International Atomic Energy Agency, Calibration of Brachy Therapy Sources 1079 (1999).

[14] R. Nath, L. L. Anderson, J. A. Meli, A. J. Olch, J. A. Stitt, and J. F. Williamson, Medical Physics 24, 1557 (1997).

[15] H. Takeda and D. Dowell, Nuclear Instruments Methods and Physics Research A 331, 384 (1993). 
[16] D. C. Jolly, Physics Letters 107A, 231 (1985).

[17] A. E. D. Heylen and K. A. Bunting, International Journal of Electronics 31, 9 (1971).

[18] H. K. Oh, Proceedings of the Optical Society of Korea Annual Meeting 2001, 124 (2001).
[19] S.-C. Zhang and J. N. Elgin, Physics Letters A 198, 89 (1995).

[20] M. T. Kim, H. K. Lee, Y. C. Heo, and J. H. Cho, J. Magn. 19, 15 (2014). 